\title{
MicroRNA-513a-3p regulates colorectal cancer cell metabolism via targeting hexokinase 2
}

\author{
CHEN LI ${ }^{1}$, ZHIJIN YU ${ }^{2}$ and JINPENG YE ${ }^{1}$ \\ ${ }^{1}$ Department of Digestive Endocrine, People's Hospital of Boluo County, Huizhou, Guangdong 516100; \\ ${ }^{2}$ Department of Gastroenterology, Huizhou Municipal Central Hospital, Huizhou, Guangdong 516002, P.R. China
}

Received August 1, 2019; Accepted November 5, 2019

DOI: $10.3892 / \mathrm{etm} .2020 .8727$

\begin{abstract}
Disruption of cell metabolism is a hallmark of cancer cells. Accumulating evidence suggests that microRNAs (miRNAs/miRs) are involved in almost all physiological and pathological processes. The aberrant expression of miRNAs induces metabolic reprogramming in cancer cells and thus, promotes proliferation. In the current study, miR-513a-3p was identified as a significantly downregulated miRNA in colorectal cancer cells and tumors. Overexpression of miR-513a-3p in colorectal cancer cells inhibited proliferation and glycolysis. A well-documented metabolic regulator, hexokinase 2 (HK2), was predicted and validated HK2 to be a target gene of miR-513a-3p in colorectal cancer cells. In addition, overexpression of HK2 reversed the miR-513a-3p mimic-induced inhibition of proliferation. The association between HK2 and miR-513a-3p was further observed in tumors collected from patients with colorectal cancer. The findings suggest that miR-513a-5p may inhibit glycolysis in colorectal cancer cells via repressing HK2 expression, indicating that miR-513a-5p may be a tumor suppressor in colorectal cancer.
\end{abstract}

\section{Introduction}

Colorectal cancer is a common type of cancer worldwide; according to global cancer statistics, the number of new cases of cancer originating from the colon and rectum reached $\sim 1,800,000$ in 2018 (1). Despite advances in our understanding of the molecular mechanism and the development of new therapeutic methods, the prognosis of patients with colorectal cancer remains poor, as the five-year overall survival is near $60 \%$ (2). This is due to the aggressive nature of colorectal cancer cells $(3,4)$. To overcome obstacles in the treatment of colorectal cancer, recent studies have shifted their focus to the

Correspondence to: $\mathrm{Dr}$ Chen Li, Department of Digestive Endocrine, People's Hospital of Boluo County, 16 Qiaoxiliulu, Huizhou, Guangdong 516100, P.R. China

E-mail: lichenblph@yeah.net

Key words: microRNA-513a-3p, colorectal cancer, hexokinase 2 metabolism of tumor cells that are pivotal for the survival and development of drug resistance of colorectal cancer cells (5-7).

Cancer cells exhibit a strong requirement for energy to sustain rapid proliferation and cell division (8). Unlike normal cells, most cancer cells predominantly obtain energy via a high rate of glycolysis even in aerobic conditions $(9,10)$. During the pathogenesis of cancer, reprogramming of metabolism leads to increased glucose consumption and lactic acid fermentation, known as Warburg effect (9). Hexokinase 2 (HK2) is a member of the HK family of enzymes that converts glucose to glucose-6-phosphate in cells (11). Elevation of HK2 activity is commonly observed in cancer cells exhibiting Warburg glycolysis (12). Glycolysis is also required for colorectal cancer cells to activate signaling pathways and maintain cell growth (13). In colorectal cancer, studies found that HK2 was upregulated in colorectal cancer cells, and its expression was associated with worse overall survival of patients with colorectal cancer (14). Most recently, targeted therapy against HK2 has shown beneficial effects on inhibiting the proliferation of colorectal cancer cells (15).

MicroRNAs (miRNAs/miRs) are small non-coding single-stranded molecules in cells (16). Mechanistically, miRNAs directly bind to the 3'untranslated region (3'UTR) region in the mRNA of genes, which leads to degradation of mRNA or inhibition of translation (17). Through the tight control of target gene expression, miRNAs are involved in almost all physiological processes (18). Dysregulation of miRNAs is associated with various types of cancer (19). Several differentially expressed miRNAs have been identified in colorectal cancer $(20,21)$. miRNAs such as miR-16, miR-17 and $\mathrm{miR}-181 \mathrm{a}$ have been experimentally proven to act as oncogenes or tumor suppressors in colorectal cancer cells (22-24). miR-1, miR-133b, miR-124, miR-137 and miR-340 have been identified as tumor suppressors via repressing the cellular metabolism of colorectal cancer $(6,25)$.

In the current study, the expression of miR-513a-3p in colorectal cancer cells and tissues was investigated. Our data suggested that miR-513a-5p inhibited glycolysis in colorectal cancer cells via repressing HK2, indicating miR-513a-5p as a tumor suppressor in colorectal cancer.

\section{Materials and methods}

Tissue collection. A total of 30 colorectal tumors and matched normal tissues were collected from patients during surgery 
in the People's Hospital of Boluo County (Huizhou, China) between February 2015 and May 2017. All participants did not receive chemotherapy or radiotherapy before surgery. Written informed consent was provided by all patients, and the protocol of current study was approved by the Ethics committee of the People's Hospital of Boluo County. The samples were immediately stored in $-80^{\circ} \mathrm{C}$ before being subjected to RNA extraction.

Cell culture. A normal colonic mucosal cell line, FHC, and colorectal cancer cell lines, HCT116 and SW480, were purchased from ATCC. Cells were cultured in DMEM medium (Invitrogen; Thermo Fisher Scientific, Inc.) supplemented with 10\% FBS (Gibco; Thermo Fisher Scientific, Inc.) in an incubator at $37^{\circ} \mathrm{C}$ with $5 \% \mathrm{CO}_{2}$.

$R N A$ isolation and RT- $q P C R$. Total RNA was extracted from tissues and cells with TRIzol reagent (Invitrogen; Thermo Fisher Scientific, Inc.) following manufacturer's protocol. The quantity and quality of RNA was determined using a NanoDrop 2000 (Thermo Fisher Scientific, Inc.). RNA was then reverse transcribed into first-stranded cDNA with PrimeScript $^{\mathrm{TM}}$ RT Reagent kit (Takara Bio, Inc.). qPCR was performed on a Real-time PCR system (Applied Biosystems; Thermo Fisher Scientific, Inc.) with a SYBR Green PCR kit (Takara Bio, Inc.) following the manufacturer's protocol (26). The quantification of miRNA was performed with the stem-loop RT-PCR as reported before (27). The RT-qPCR condition was: predenature at $95^{\circ} \mathrm{C}$ for $30 \mathrm{sec}$, followed by 35 cycles of denature at $95^{\circ} \mathrm{C}$ for $10 \mathrm{sec}$ and elongation at $55^{\circ} \mathrm{C}$ for $20 \mathrm{sec} . \beta$-actin and U6 were used as internal controls for mRNA and miRNA, respectively. The primer sequences were as follows: $\beta$-actin forward, 5'-CATGTACGTTGC TATCCAGGC-3' and reverse, 5'-CTCCTTAATGTCACG CACGAT-3'; HK2 forward, 5'-GAGCCACCACTCACCCTA CT-3' and reverse, 5'-CCAGGCATTCGGCAATGTG-3'; enolase 2 (ENO2) forward, 5'-AGCCTCTACGGGCATCTA TGA-3' and reverse, 5'-TTCTCAGTCCCATCCAACTCC-3'; phosphoglycerate kinase 1 (PGK1) forward, 5'-TGGACG TTAAAGGGAAGCGG-3' and reverse, 5'-GCTCATAAG GACTACCGACTTGG-3'; phosphofructokinase platelet (PFKP) forward, 5'-GCATGGGTATCTACGTGGGG-3' and reverse, 5'-CTCTGCGATGTTTGAGCCTC-3'; hexokinase 1 (HK1) forward, 5'-GCTCTCCGATGAAACTCTCAT AG-3' and reverse, 5'-GGACCTTACGAATGTTGGCAA-3'; aldolase A (ALDOA) forward, 5'-ATGCCCTACCAATAT CCAGCA-3' and reverse, 5'-GCTCCCAGTGGACTCATC TG-3'; miR-513a-3p forward, 5'-GCCGAGTTTTAATTT ATATT-3' and reverse, 5'-CTCAACTGGTGTCGTGGA-3'; and U6 forward, 5'-GCGCGTCGTGAAGCGTTC-3' and reverse, 5'-GTGCAGGGTCCGAGGT-3'. The sequence for the stem-loop primer was 5'-CTCAACTGGTGTCGTGGA GTCGGCAATTCAGTTGAGAAAATT-3'.

Protein isolation and western blotting. Total protein was extracted from cells using RIPA lysis buffer (Thermo Fisher Scientific, Inc.) according to the manufacturer's guidelines. After preparation of lysates, the concentration was determined with a bicinchoninic acid kit (Pierce; Thermo Fisher Scientific, Inc.). A total of $20 \mu \mathrm{g}$ of each sample was electrophoresed on an $8 \%$ gel using SDS-PAGE, and proteins were transferred to a PVDF membrane. The membrane was incubated in primary antibodies and secondary antibodies for $1 \mathrm{~h}$ at room temperature sequentially. The blots were detected and visualized by a chemiluminescence detection system (Pierce; Thermo Fisher Scientific, Inc.). Primary antibodies against $\operatorname{HK} 2$ (1:2,000; cat. no. ab228819) and $\beta$-actin (1:10,000; cat. no. ab8226,) were purchased from Abcam. HRP-conjugated secondary antibodies against mouse (1:50,000; cat. no. SA00001-1) and rabbit (1:50,000; cat. no. SA00001-2) were obtained from ProteinTech Group, Inc.

Overexpression of miR-513a-3p. The miR-513a-3p mimic (5'-UAAAUUUCACCUUUCUGAGAAGG-3') and negative control (miR-NC) mimic (5'-AUUGGAACGAUACAGAGA AGAUU-3') were synthesized and purchased from Shanghai GenePharma Co., Ltd. 1×10 ${ }^{6}$ HCT116 or SW480 cells were transfected with $50 \mathrm{nM}$ miR-513a-3p mimic or miR-NC mimic using Lipofectamine 3000 (Invitrogen; Thermo Fisher Scientific, Inc.) according to the manufacturer's protocol. At $48 \mathrm{~h}$ after transfection, cells were harvested for subsequent experiments.

Overexpression of HK2. Overexpression of HK2 was achieved via transfection of the pcDNA3 plasmid containing full length cDNA of HK2. The cDNA of HCT116 was prepared as aforementioned. The full length of HK2 was amplified from HCT116 cDNA and ligated into the pcDNA3 plasmid (Addgene, Inc.). The primer sequences were: HK2-forward: 5'-AAGCTTATGATTGCCTCGCA-3'; HK2-reverse: 5'- TCT AGACTATCGCTGTC-3'. A total of $2 \mu \mathrm{g}$ pcDNA3-HK2 or pcDNA3 was co-transfected with $50 \mathrm{nM}$ miR-NC mimic or miR-513a-3p mimic into 1 X10 ${ }^{6}$ HCT116 or SW480 cells with Lipofectamine 3000 . After $48 \mathrm{~h}$, cells were subjected to subsequent experiments.

Determination of cell proliferation. The proliferation ability of cells was detected with a Cell Counting Kit-8 (CCK-8) assay (Dojindo Molecular Technologies, Inc.) according to the manufacturer's protocol. In brief, 10,000 cells were plated into each well of 96-well plates. At the timepoints of 0, 24, 48 and $72 \mathrm{~h}$ after transfection, $10 \mu \mathrm{l} \mathrm{CCK}-8$ solution was added to the wells and incubated for $2 \mathrm{~h}$ at $37^{\circ} \mathrm{C}$. The medium containing CCK-8 solution was then transferred to another 96-well plate. The absorbance at $450 \mathrm{~nm}$ was detected, and was considered to reflect cell number.

Colorimetric assay of glucose uptake. The glucose uptake ability of HCT116 and SW480 cells was determined with a Glucose Uptake Colorimetric Assay kit (BioVision, Inc.) following manufacturers' protocol. A total of 2,000 cells were plated in each well of 96-well plates. Cells were transfected with miR-NC mimic or miR-513a-3p mimic, as aforementioned. After $24 \mathrm{~h}$, the cells were starved for glucose by preincubating with $100 \mu \mathrm{l}$ Krebs-Ringer-Phosphate-HEPES buffer containing $2 \% \mathrm{BSA}$ for $40 \mathrm{~min}$ at $37^{\circ} \mathrm{C}$. Next, $10 \mu \mathrm{l}$ $10 \mathrm{mM} 2$-deoxy-D-glucose was added and the cells were incubated for $20 \mathrm{~min}$. The absorbance at $412 \mathrm{~nm}$ was detected every 5 mins until the $100 \mathrm{pM}$ standard reached 1.5 . 

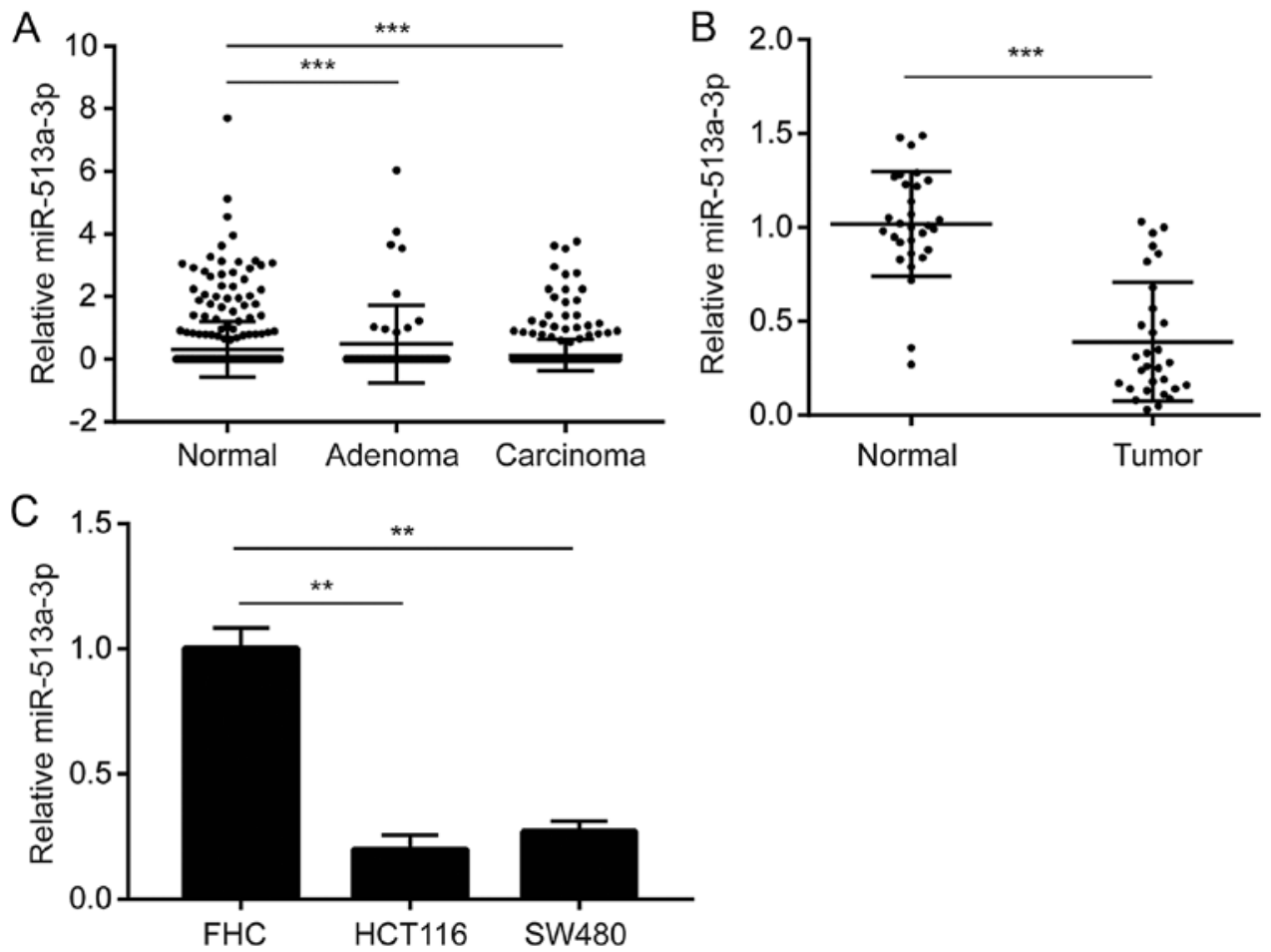

Figure 1. miR-513a-3p was decreased in colorectal cancer. (A) Analysis of GSE115513 dataset showed that miR-513a-3p levels were decreased in colon adenoma and carcinoma compared to colon mucosa. (B) RT-qPCR showed that miR-513a-3p was significantly decreased in tumors compared to matched normal tissues from patients with colorectal cancer. (C) RT-qPCR showed that miR-513a-3p levels were also lower in colorectal cancer cell lines (HCT116 and SW480) compared to the immortal colorectal cell line (FHC). ${ }^{* * *} \mathrm{P}<0.01 ;{ }^{* * * *} \mathrm{P}<0.001$.

Detection of lactate levels. The lactate levels in the culture medium were detected using a Lactate Colorimetric/Fluorometric Assay kit (BioVision, Inc.) following the manufacturer's protocol. A total of 2,000 cells were seeded in each well of 96-well plates and transfected with miR-513a-3p mimic or miR-NC mimic. After $48 \mathrm{~h}$, lactate assay buffer was added to each well, and after incubation for 30 mins, absorbance at $570 \mathrm{~nm}$ was detected and was considered to reflect lactate levels.

Bioinformatic analysis and dual luciferase reporter assay. Using GEO database (https://www.ncbi.nlm.nih.gov/gds/), the differentially expression miRNA in colon adenoma, colon carcinoma and normal tissues were analyzed based on GSE115513 (including expression profile of 381 normal tissues, 51 colon adenoma and 411 colon carcinoma). The potential target genes and putative binding sites for miR-513a-3p were analyzed with TargetScan software (www.targetscan.org/vert_72/). The 3'UTR containing the putative binding sites for miR-513-3p was cloned using HCT116 cDNA and ligated into the pGL3 plasmid (Promega Corporation). The primer sequence: HK2-forward: 5'-CTC TAGAAACCCCTGAAATCG-3'; HK2-reverse: 5'-CTC TAGATTTGATTATTTTGGA-3'. A total of $1 \times 10^{5}$ cells were seeded into each well in 24-well plates. Cells were transfected with $2 \mu \mathrm{g}$ pGL3-HK2-3'UTR-WT or pGL3-HK2-3'UTR-Mut and miR-513a-3p mimic or miR-NC mimic using Lipofectamine 3000. At $24 \mathrm{~h}$ after transfection, the relative luciferase activity was detected using a Dual Luciferase Reporter Assay system (Promega Corporation).
Statistical analysis. All data were calculated with GraphPad Prism 6 (GraphPad Software, Inc.) and presented as mean \pm SD. Differences between two groups were analyzed using a two-tailed Student's t-test. Differences between multiple groups were assessed using one-way ANOVA followed by the Dunnett's post hoc test. Pearson's correlation analysis was used to analyze the correlation between miR-513a-3p expression and HK2 mRNA expression levels. Each experiment was repeated at least 3 times. $\mathrm{P}<0.05$ was considered to indicate a statistically significant difference.

\section{Results}

Downregulation of miR-513a-3p in colorectal cancer. miRNA expression data of colorectal cancer was retrieved from published datasets. miR-513a-3p was found to be significantly downregulated in colon adenoma and colon carcinoma compared with normal colon tissues (Fig. 1A). For validation, 30 pairs of tumor and normal tissues were collected from patients with colorectal cancer. RT-qPCR results suggested that miR-513a-3p was downregulated in colorectal tumors (Fig. 1B). In addition, miR-513a-3p levels were also lower in the colorectal cancer cell lines HCT116 and SW480 compared with the normal colonic mucosal cell line, FHC (Fig. 1C).

Overexpression of miR-513a-3p suppresses colorectal cancer cell proliferation and metabolism. To investigate the role of miR-513a-3p in colorectal cancer cells, miR-513a-3p mimic was transfected into HCT116 and SW480 cells. Transfection of miR-513a-3p mimic induced a 10-fold increase in the expression of miR-513a-3p in HCT116 cells 

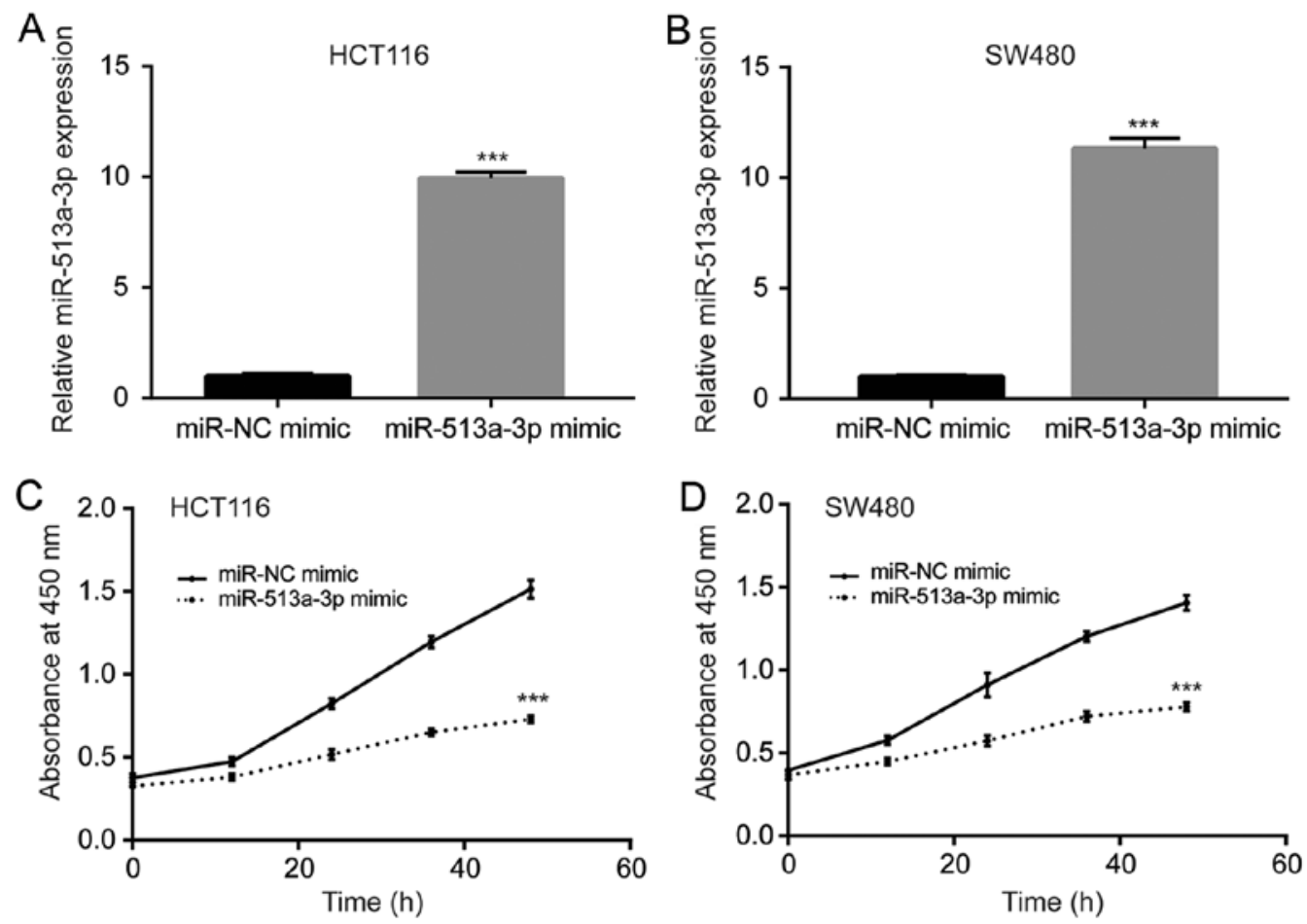

Figure 2. MiR-513a-3p inhibited colorectal cancer cell proliferation. (A) Transfection of miR-513a-3p mimic elevated miR-513a-3p expression in HCT116 cells. (B) Transfection of miR-513a-3p mimic elevated miR-513a-3p expression in SW480 cells. (C) Overexpression of miR-513a-3p reduced cell proliferation of HCT116 cells. (D) Overexpression of miR-513a-3p reduced cell proliferation of SW480 cells. ${ }^{* * * *} \mathrm{P}<0.001$.
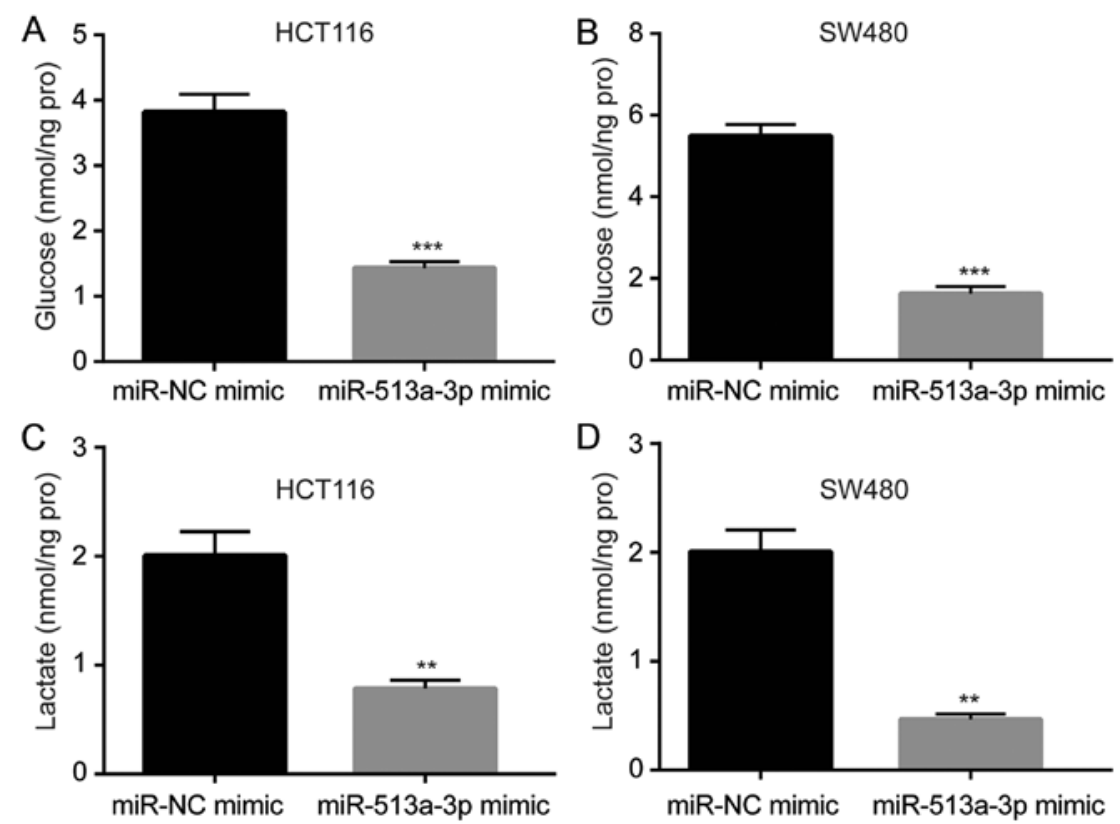

Figure 3. MiR-513a-3p inhibited glycolysis of colorectal cancer cells. (A) Overexpression of miR-513a-3p inhibited glucose uptake of HCT116 cells (B) Overexpression of miR-513a-3p inhibited glucose uptake of SW480 cells. (C) Overexpression of miR-513a-3p decreased lactate levels in culture medium of HCT116 cells. (D) Overexpression of miR-513a-3p decreased lactate levels in culture medium of SW480 cells. ${ }^{* *} \mathrm{P}<0.01 ;{ }^{* * * *} \mathrm{P}<0.001$.

(Fig. 2A). Similarly, miR-513a-3p expression also increased in SW480 cells after transfection with miR-513a-3p mimic (Fig. 2B). The proliferation of colorectal cancer cells was detected with a CCK-8 kit. It was found that miR-513a-3p overexpression significantly reduced proliferation of HCT116 cells (Fig. 2C). In SW480 cells, overexpression of miR-513a-3p also inhibited proliferation (Fig. 2D). Increased proliferation of colorectal cancer cells is associated with aberrant cell metabolism, including an abnormally high rate of glycolysis. miR-513a-3p overexpression significantly reduced the glucose uptake rate in HCT116 and SW480 cells (Fig. 3A and B). Additionally, lactate levels in the supernatant of cultured cells were significantly decreased after miR-513a-3p overexpression (Fig. 3C and D). These 

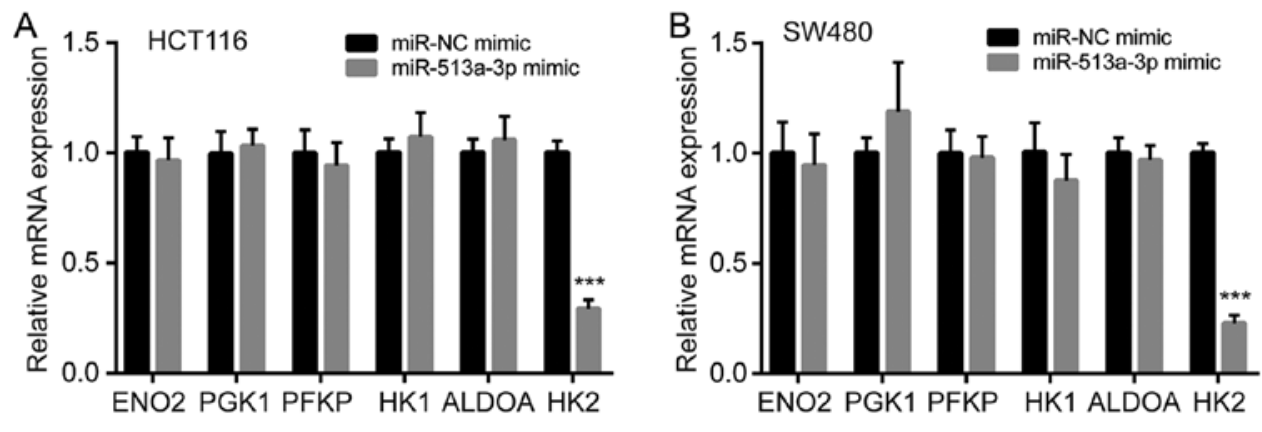
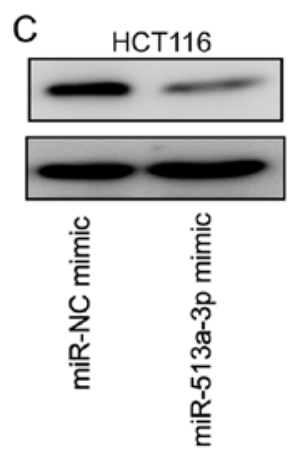
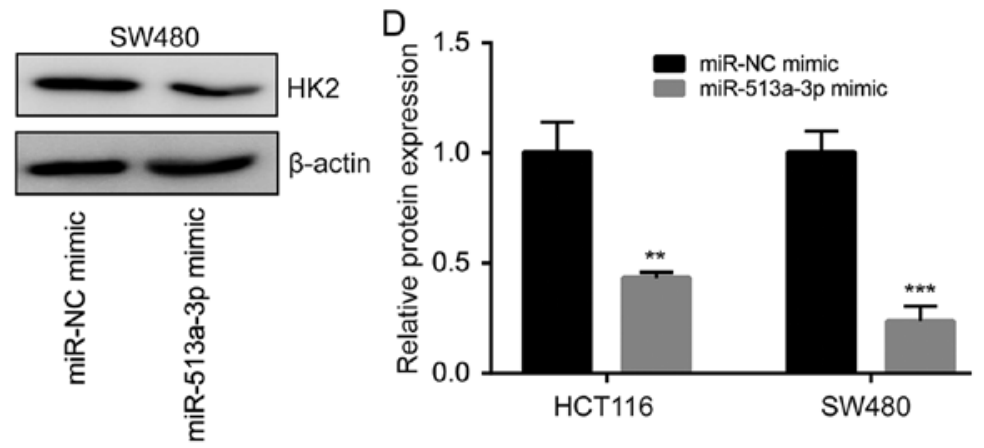

Figure 4. MiR-513a-3p decreased HK2 expression in colorectal cancer cells. (A) Among a panel of glycolysis associated genes, miR-513a-3p overexpression decreased HK2 mRNA levels in HCT116 cells. (B) Among a panel of glycolysis associated genes, miR-513a-3p overexpression decreased HK2 mRNA levels in SW480 cells. (C) Overexpression of miR-513a-3p decreased HK2 protein expression in HCT116 and SW480 cells. (D) Quantitative analysis of HK2 protein expression in $\mathrm{C} .{ }^{* *} \mathrm{P}<0.01 ;{ }^{* * *} \mathrm{P}<0.001$.

A

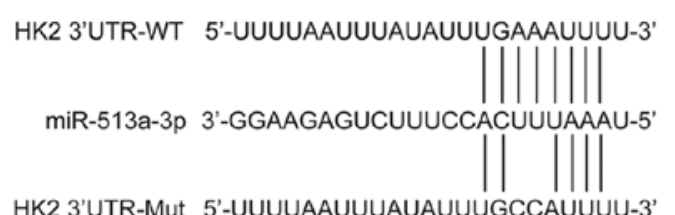

C

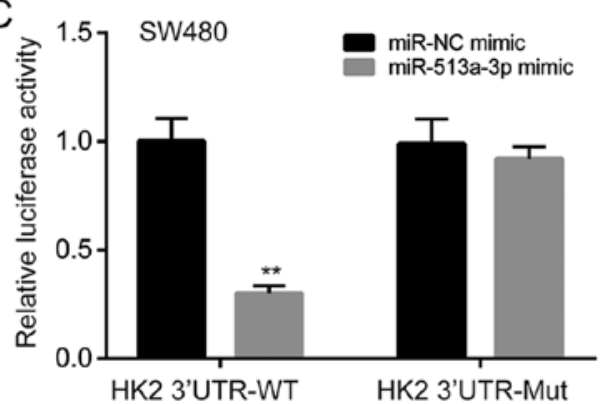

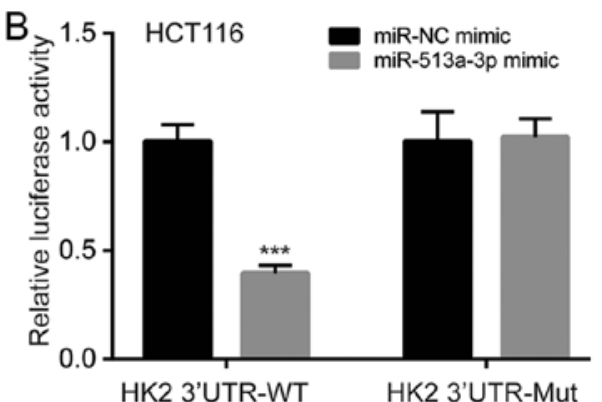

HK2 3'UTR-WT HK2 3'UTR-Mut

Figure 5. MiR-513a-3p directly targeted HK2. (A) The sequence alignment showed that miR-513a-3p might bind to 3'UTR of HK2 mRNA. The sequence of mutant type of HK2 3'UTR was also showed. (B) In the dual luciferase reporter assay, miR-513a-3p overexpression decreased relative luciferase activity in HCT116 cells transfected with HK2 3'UTR-WT. (C) In the dual luciferase reporter assay, miR-513a-3p overexpression decreased relative luciferase activity in SW480 cells transfected with HK2 3 'UTR-WT. ${ }^{* *} \mathrm{P}<0.01 ;{ }^{* * *} \mathrm{P}<0.001$.

results suggested that miR-513a-3p negatively regulated the glycolytic process of colorectal cancer cells to inhibit proliferation.

miR-513a-3p inhibits HK2 expression in colorectal cancer cells. To investigate how miR-513a-3p regulates glycolysis in colorectal cancer cells, RT-qPCR was performed to detect the expression of multiple key genes involved in glycolysis, including ENO2, PGK1, PFKP, HK1, ALDOA and
HK2 $(28,29)$. The results showed that mRNA expression of HK2 was significantly reduced while the expression of ENO2, PGK1, PFKP, HK1 and ALDOA was not changed in HCT116 cells transfected with miR-513a-3p mimic (Fig. 4A). In SW480 cells, the decrease of HK2 mRNA expression was also observed following miR-513a-3p overexpression (Fig. 4B). Additionally, western blotting showed that miR-513a-3p overexpression decreased HK2 protein expression in both HCT116 and SW480 cells (Fig. 4C and D). 
A

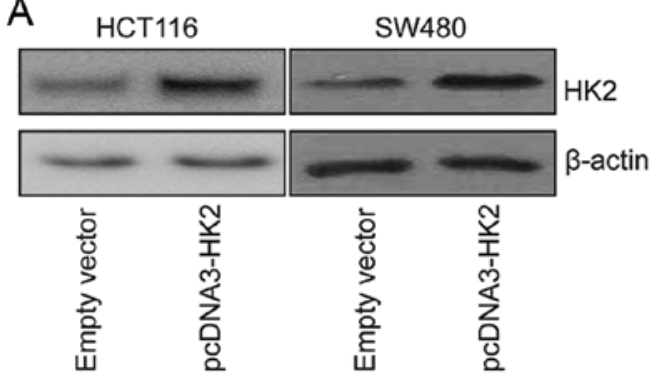

C

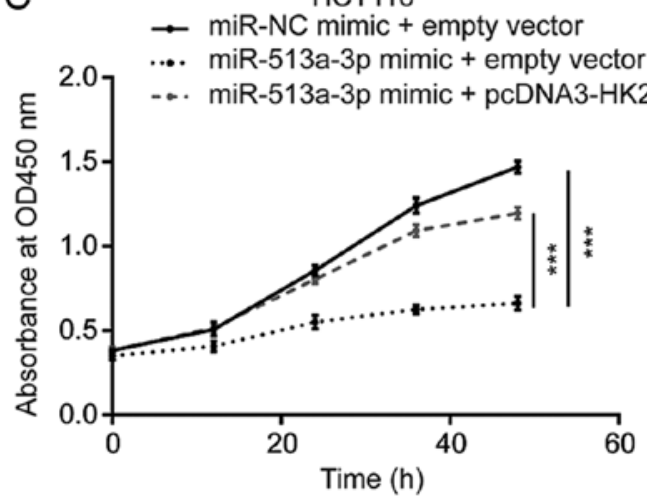

E

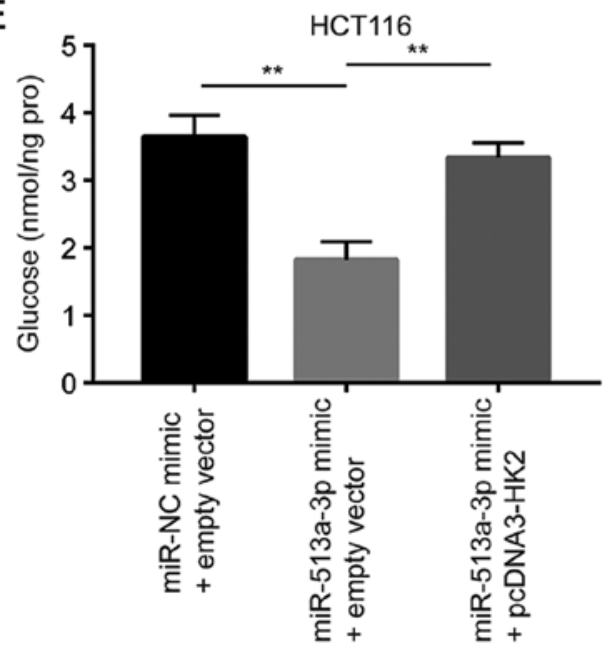

G

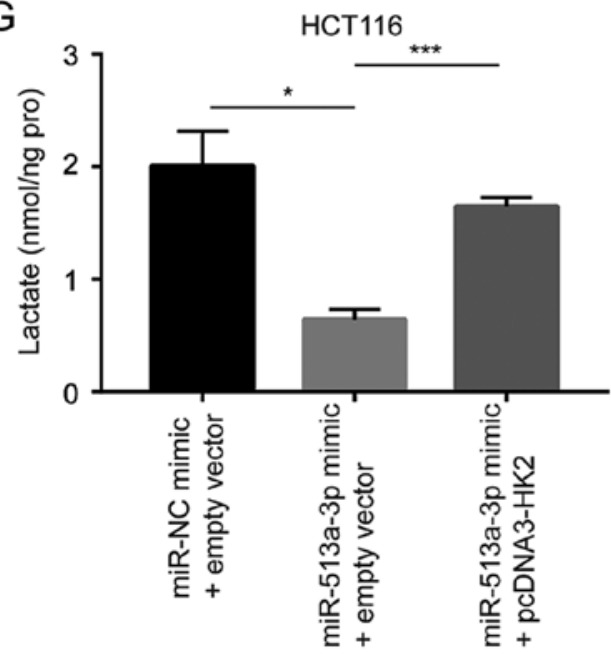

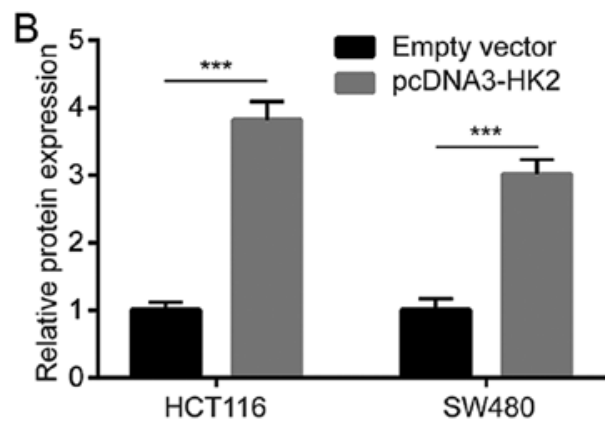

SW480

D

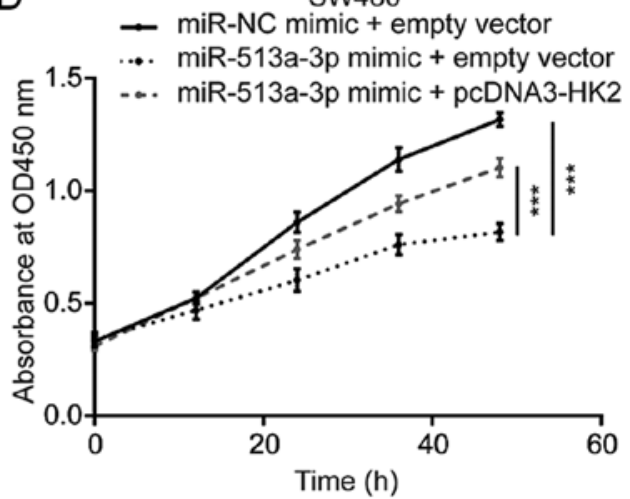

$\mathrm{F}$

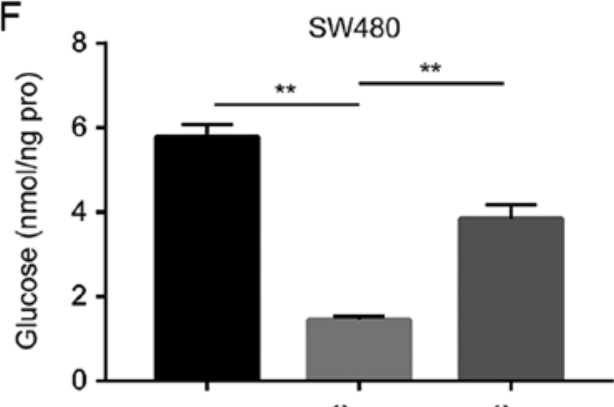

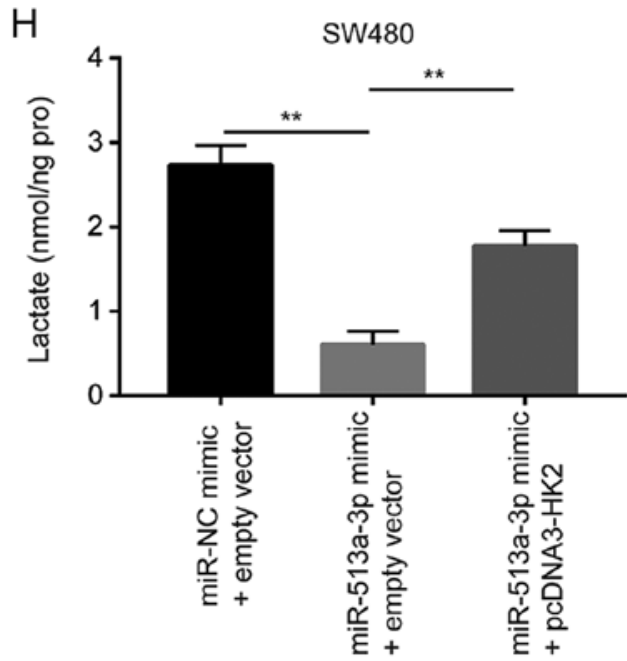

Figure 6. HK2 was pivotal for regulation of cell proliferation and glycolysis by miR-513a-3p in colorectal cancer cells. (A) Western blotting showed that transfection of pcDNA3-HK2 elevated HK2 protein expression in HCT116 and SW480 cells. (B) Quantitative analysis of HK2 expression in A. (C and D) The cell proliferation assay showed that the overexpression of HK2 reversed cell proliferation inhibition led by miR-513a-3p mimic in HCT116 and SW480 cells. (E and F) Overexpression of HK2 reversed reduction of glucose uptake induced by miR-513a-3p mimic in HCT116 and SW480 cells. (G and H) Overexpression of HK2 reversed reduction of lactate levels induced by miR-513a-3p mimic in culture medium of $\mathrm{HCT} 116$ and $\mathrm{SW} 480$ cells. ${ }^{*} \mathrm{P}<0.05$; ${ }^{* *} \mathrm{P}<0.01$; ${ }^{* * *} \mathrm{P}<0.001$. 

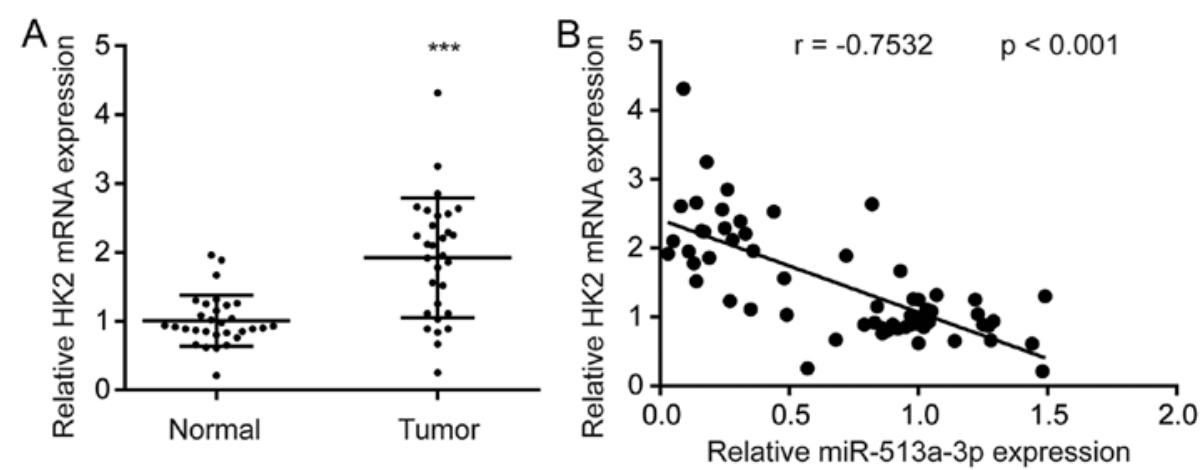

Figure 7. HK2 mRNA levels were negatively correlated with miR-513a-3p levels in tumors and normal tissues from colorectal cancer patients. (A) RT-qPCR showed that HK2 mRNA expression was elevated in tumors compared with matched normal tissues from 30 patients with colorectal cancer. (B) The Pearson correlation analysis suggested that miR-513a-3p levels were negatively correlated with HK2 mRNA expression in tumors and normal tissues from 30 patients with colorectal cancer. ${ }^{* * *} \mathrm{P}<0.001$.

miR-513a-3p directly represses HK2 expression. To explore whether miR-513a-3p directly regulated HK2 expression, bioinformatic analysis was performed. It was found that there was a putative binding site for miR-513a-3p in the $3^{\prime} \mathrm{UTR}$ of HK2 mRNA (Fig. 5A). A luciferase reporter plasmid containing wild-type (HK2 3'UTR-WT) or mutant (HK2 3'UTR-Mut) 3'UTR of the HK2 mRNA was constructed (Fig. 5A). The dual luciferase reporter assay showed that miR-513a-3p overexpression reduced relative luciferase activity in HCT116 cells transfected with HK2 3'UTR-WT, but not in cells transfected with HK2 3'UTR-Mut (Fig. 5B). The results in HCT116 cells were consistent with results in SW480 cells, as the miR-513a-3p mimic also reduced relative luciferase activity in SW480 cells transfected with HK2 3'UTR-WT only (Fig. 5C). These results indicated that miR-513a-3p directly bound to HK2 mRNA to repress its expression in colorectal cancer cells.

miR-513a-3p inhibits proliferation and glycolysis of colorectal cancer cells via targeting $H K 2$. A plasmid containing the HK2 cDNA sequence was constructed. Transfection of pcDNA3-HK2 upregulated HK2 protein expression in HCT116 and SW480 cells (Fig. 6A and B). A proliferation assay revealed that overexpression of HK2 reversed the reduction of proliferation induced by miR-513a-3p mimic in HCT116 and SW480 cells (Fig. 6C and D). Additionally, the decrease of glucose uptake induced by miR-513a-3p mimic was also reversed after HK2 overexpression in HCT116 and SW480 cells (Fig. 6E and F). The lactate levels recovered after HK2 overexpression in HCT116 and SW480 cells treated with miR-513a-3p mimic (Fig. 6G and H).

Expression of miR-513a-3p is negatively correlated with HK2 mRNA expression in colorectal tumors and normal tissues. To examine the clinical association between miR-513a-3p and HK2, HK2 mRNA expression was detected in colorectal tumors and normal tissues. HK2 mRNA levels were significantly upregulated in colorectal tumors compared with normal tissues (Fig. 7A). Pearson correlation analysis suggested that miR-513a-3p levels were negatively correlated with HK2 mRNA expression in tumors and normal tissues from patients with colorectal cancer (Fig. 7B).

\section{Discussion}

Numerous studies have demonstrated that miRNAs are pivotal for colorectal cancer progression and may be used as therapeutic targets and biomarkers for patients with colorectal cancer $(23,24,30)$. Several studies indicated that miR-513a-3p was involved in carcinogenesis $(31,32)$. Zhang et al (31) found that miR-513a-3p was upregulated in cisplatin-resistant lung cancer cells, and that it sensitized lung cancer cells to cisplatin treatment via targeting GSTP1. Another study showed that miR-513a-3p regulated the inflammatory process and the migration of lung cancer cells through direct repression of Integrin Beta-8 (32). The results of the present study suggest an antitumor role for miR-513a-3p in colorectal cancer. miR-513a-3p was found to be downregulated in colorectal cancer via bioinformatic analysis and RT-qPCR validation. In two colorectal cancer cell lines, overexpression of miR-513a-3p inhibited proliferation. As abnormal metabolism is essential for sustained proliferative signaling in colorectal cancer cells (6), glycolysis was examined in colorectal cancer cells transfected with miR-513a-3p mimic. Interestingly, the glucose uptake rate was suppressed following miR-513a-3p overexpression. Additionally, the lactate level in the culture medium of cells with miR-513a-3p overexpression was decreased. The present study demonstrated that miR-513a-3p influenced the metabolism and proliferation of colorectal cancer cells.

The aberrant activation of metabolism in cancer cells is the consequence of promoter methylation, gene mutation and altered expression of non-coding RNA $(10,33)$. In hepatocellular carcinoma, the promoter of HK2 is hypomethylated, resulting in enhanced HK2 expression (34). HK2 is essential for initiation of KRAS mutation-driven tumors in mouse (35). In KRAS mutant colorectal cancer cells (HCT116 and DLD-1), HK2 expression was significantly higher compared with colorectal cancer cells with wild type KRAS (36). In colon and hepatocellular cancer cells, miRNAs such as miR-98 and miR-125 were found to be negative regulators of glycolysis via targeting HK2 $(37,38)$. In the current study, several key regulators of glycolysis were screened in cells overexpressing miR-513a-3p. HK2 was downregulated after miR-513a-3p overexpression in HCT116 and SW480 cells, two KRAS-mutant colorectal cancer cell lines. miR-513a-3p 
was predicted and validated to be a novel direct regulator of HK2 in cancer cells. HK2 is pivotal for cell proliferation, stemness, metabolism and drug resistance of colorectal cancer cells $(39,40)$. In the current study, HK2 overexpression reversed the inhibition of cell proliferation caused by miR-513a-3p overexpression. Furthermore, HK2 overexpression enhanced glucose uptake and elevated lactate levels, which were inhibited by miR-513a-3p overexpression. The results of this study suggest that miR-513a-3p/HK2 interaction is a driver of glycolysis and cell proliferation in colorectal cancer.

In summary, the present study explored the role of miR-513a-3p in colorectal cancer. Downregulation of miR-513a-3p directly increased HK2 expression and promoted proliferation and metabolism in colorectal cancer cells. These findings may serve as rationale for targeting miR-513a-3p and HK2 as a novel therapeutic approach for patients with colorectal cancer.

\section{Acknowledgements}

Not applicable.

\section{Funding}

No funding was received.

\section{Availability of data and materials}

The datasets used and/or analyzed during the present study are available from the corresponding author on reasonable request.

\section{Authors' contributions}

JY conceived and designed the present study. CL, ZY and JY performed the experiments. CL and ZY coordinated the research and analyzed the data. JY wrote the manuscript and supervised the study. All of the authors received the final version of manuscript and approved for publication.

\section{Ethics approval and consent to participate}

The current study was approved by the Ethical Committee of the People's Hospital of Boluo County (Huizhou, China). Each patient provided written informed consent prior to the study.

\section{Patient consent for publication}

Not applicable.

\section{Competing interests}

The authors declare that they have no competing interests.

\section{References}

1. Bray F, Ferlay J, Soerjomataram I, Siegel RL, Torre LA and Jemal A: Global cancer statistics 2018: GLOBOCAN estimates of incidence and mortality worldwide for 36 cancers in 185 countries. CA Cancer J Clin 68: 394-424, 2018.
2. Moghimi-Dehkordi B and Safaee A: An overview of colorectal cancer survival rates and prognosis in Asia. World J Gastro Oncol 4: 71-75, 2012.

3. Siegel R, Desantis C and Jemal A: Colorectal cancer statistics, 2014. CA Cancer J Clin 64: 104-117, 2014.

4. Hayat MJ, Howlader N, Reichman ME and Edwards BK: Cancer statistics, trends, and multiple primary cancer analyses from the surveillance, epidemiology, and end results (SEER) Program. Oncologist 12: 20-37, 2007.

5. Zhang M, Liu T, Sun H, Weng W, Zhang Q, Liu C, Han Y and Sheng W: Pim1 supports human colorectal cancer growth during glucose deprivation by enhancing the Warburg effect. Cancer Sci 109: 1468-1479, 2018.

6. Taniguchi K, Sakai M, Sugito N, Kumazaki M, Shinohara H, Yamada N, Nakayama T, Ueda H, Nakagawa Y, Ito Y, et al: PTBP1-associated microRNA-1 and -133 b suppress the Warburg effect in colorectal tumors. Oncotarget 7: 18940-18952, 2016.

7. Nijhuis A, Thompson H, Adam J, Parker A, Gammon L, Lewis A, Bundy JG, Soga T, Jalaly A, Propper D, et al: Remodelling of microRNAs in colorectal cancer by hypoxia alters metabolism profiles and 5-fluorouracil resistance. Hum Mol Genet 26: 1552-1564, 2017.

8. Warburg O: On the origin of cancer cells. Science 123: 309-314, 1956.

9. Hsu PP and Sabatini DM: Cancer cell metabolism: Warburg and beyond. Cell 134: 703-707, 2008.

10. Vander Heiden MG, Cantley LC and Thompson CB: Understanding the Warburg effect: The metabolic requirements of cell proliferation. Science 324: 1029-1033, 2009.

11. Vartanian A, Agnihotri S, Wilson MR, Burrell KE, Tonge PD, Alamsahebpour A, Jalali S, Taccone MS, Mansouri S, Golbourn B, et al: Targeting hexokinase 2 enhances response to radio-chemotherapy in glioblastoma. Oncotarget 7: 69518-69535, 2016.

12. Jin Z, Gu J, Xin X, Li Y and Wang H: Expression of hexokinase 2 in epithelial ovarian tumors and its clinical significance in serous ovarian cancer. Eur J Gynaecol Oncol 35: 519-524, 2014.

13. Graziano F, Ruzzo A, Giacomini E, Ricciardi T, Aprile G, Loupakis F, Lorenzini P, Ongaro E, Zoratto F, Catalano V, et al: Glycolysis gene expression analysis and selective metabolic advantage in the clinical progression of colorectal cancer. Pharmacogenomics J 17: 258-264, 2017.

14. Katagiri M, Karasawa H, Takagi K, Nakayama S, Yabuuchi S, Fujishima F, Naitoh T, Watanabe M, Suzuki T, Unno M and Sasano H: Hexokinase 2 in colorectal cancer: A potent prognostic factor associated with glycolysis, proliferation and migration. Histol Histopathol 32: 351-360, 2017.

15. Kudryavtseva AV, Fedorova MS, Zhavoronkov A, Moskalev AA, Zasedatelev AS, Dmitriev AA, Sadritdinova AF, Karpova IY, Nyushko KM, et al: Effect of lentivirus-mediated shRNA inactivation of HK1, HK2, and HK3 genes in colorectal cancer and melanoma cells. BMC Genet 17 (Suppl 3): S156, 2016.

16. Alvarez-Garcia I and Miska EA: MicroRNA functions in animal development and human disease. Development 132: 4653-4662, 2005.

17. Treiber T, Treiber N and Meister G: Regulation of microRNA biogenesis and function. Thromb Haemost 107: 605-610, 2012.

18. Zhao Y and Srivastava D: A developmental view of microRNA function. Trends Biochem Sci 32: 189-197, 2007.

19. Mo YY: MicroRNA regulatory networks and human disease. Cell Mol Life Sci 69: 3529-3531, 2012.

20. Moridikia A, Mirzaei H, Sahebkar A and Salimian J: MicroRNAs: Potential candidates for diagnosis and treatment of colorectal cancer. J Cell Physiol 233: 901-913, 2018.

21. Slattery ML, Herrick JS, Pellatt DF, Stevens JR, Mullany LE, Wolff E, Hoffman MD, Samowitz WS and Wolff RK: MicroRNA profiles in colorectal carcinomas, adenomas and normal colonic mucosa: Variations in miRNA expression and disease progression. Carcinogenesis 37: 245-261, 2016.

22. You C, Liang H, Sun W, Li J, Liu Y, Fan Q, Zhang H, Yue X, $\mathrm{Li} J$, Chen $\mathrm{X}$ and $\mathrm{Ba}$ Y: Deregulation of the miR-16-KRAS axis promotes colorectal cancer. Sci Rep 6: 37459, 2016.

23. Huang C, Liu J, Xu L, Hu W, Wang J, Wang M and Yao X: MicroRNA-17 promotes cell proliferation and migration in human colorectal cancer by downregulating SIK1. Cancer Manag Res 11: 3521-3534, 2019.

24. Zhang X, Li X, Tan F, Yu N and Pei H: STAT1 inhibits MiR-181a expression to suppress colorectal cancer cell proliferation through PTEN/Akt. J Cell Biochem 118: 3435-3443, 2017. 
25. Sun Y, Zhao X, Zhou Y and Hu Y: miR-124, miR-137 and miR-340 regulate colorectal cancer growth via inhibition of the Warburg effect. Oncol Rep 28: 1346-1352, 2012.

26. Livak KJ and Schmittgen TD: Analysis of relative gene expression data using real-time quantitative PCR and the 2(-Delta Delta C(T)) method. Methods 25: 402-408, 2001.

27. Chen C, Ridzon DA, Broomer AJ, Zhou Z, Lee DH, Nguyen JT, Barbisin M, Xu NL, Mahuvakar VR, Andersen MR, et al: Real-time quantification of microRNAs by stem-loop RT-PCR Nucleic Acids Res 33: e179, 2005.

28. Oparina NY, Snezhkina AV, Sadritdinova AF, Veselovskii VA, Dmitriev AA, Senchenko VN, Mel'nikova NV, Speranskaya AS, Darii MV, Stepanov OA, et al: Differential expression of genes that encode glycolysis enzymes in kidney and lung cancer in humans. Genetika 49: 814-823, 2013 (In Russian).

29. He Y, Deng F, Zhao S, Zhong S, Zhao J, Wang D, Chen X, Zhang J, Hou J, Zhang W, et al: Analysis of miRNA-mRNA network reveals miR-140-5p as a suppressor of breast cancer glycolysis via targeting GLUT1. Epigenomics 11: 1021-1036, 2019.

30. Markopoulos GS, Roupakia E, Tokamani M, Chavdoula E, Hatziapostolou M, Polytarchou C, Marcu KB, Papavassiliou AG, Sandaltzopoulos R and Kolettas E: A step-by-step microRNA guide to cancer development and metastasis. Cell Oncol (Dordr) 40: 303-339, 2017.

31. Zhang X, Zhu J, Xing R, Tie Y, Fu H, Zheng X and Yu B: miR-513a-3p sensitizes human lung adenocarcinoma cells to chemotherapy by targeting GSTP1. Lung Cancer 77: 488-494, 2012.

32. da Silveira MB, Lima KF, da Silva AR, Dos Santos RAS and Moraes KCM: Mir-513a-3p contributes to the controlling of cellular migration processes in the A549 lung tumor cells by modulating integrin $\beta-8$ expression. Mol Cell Biochem 444 43-52, 2018.
33. Ju HQ, Zhan G, Huang A, Sun Y, Wen S, Yang J, Lu WH, Xu RH, $\mathrm{Li} \mathrm{J}$, Li Y, et al: ITD mutation in FLT3 tyrosine kinase promotes Warburg effect and renders therapeutic sensitivity to glycolytic inhibition. Leukemia 31: 2143-2150, 2017.

34. Lee HG, Kim H, Son T, Jeong Y, Kim SU, Dong SM, Park YN, Lee JD, Lee JM and Park JH: Regulation of HK2 expression through alterations in $\mathrm{CpG}$ methylation of the HK2 promoter during progression of hepatocellular carcinoma. Oncotarget 7: 41798-41810, 2016

35. Patra KC, Wang Q, Bhaskar PT, Miller L, Wang Z, Wheaton W, Chandel N, Laakso M, Muller WJ, Allen EL, et al: Hexokinase 2 is required for tumor initiation and maintenance and its systemic deletion is therapeutic in mouse models of cancer. Cancer Cell 24: 213-228, 2013.

36. Iwamoto $\mathrm{M}$, Kawada $\mathrm{K}$, Nakamoto $\mathrm{Y}$, Itatani $\mathrm{Y}$, Inamoto $\mathrm{S}$, Toda K, Kimura H, Sasazuki T, Shirasawa S, Okuyama H, et al: Regulation of 18F-FDG accumulation in colorectal cancer cells with mutated KRAS. J Nucl Med 55: 2038-2044, 2014.

37. Zhu W, Huang Y,Pan Q, Xiang P, Xie N and Yu H: MicroRNA-98 suppress warburg effect by targeting HK2 in colon cancer cells. Dig Dis Sci 62: 660-668, 2017.

38. Jin F, Wang Y, Zhu Y, Li S, Liu Y, Chen C, Wang X, Zenz K and $\mathrm{Li} \mathrm{L}$ : The miR-125a/HK2 axis regulates cancer cell energy metabolism reprogramming in hepatocellular carcinoma. Sci Rep 7: 3089, 2017.

39. Li Q, Qin Y, Wei P, Lian P, Li Y, Xu Y, Li X, Li D and Cai S: Gas1 inhibits metastatic and metabolic phenotypes in colorectal carcinoma. Mol Cancer Res 14: 830-840, 2016.

40. Hamabe A, Yamamoto H, Konno M, Uemura M, Nishimura J, Hata T, Takemasa I, Mizushima T, Nishida N, Kawamoto K, et al: Combined evaluation of hexokinase 2 and phosphorylated pyruvate dehydrogenase-E1 $\alpha$ in invasive front lesions of colorectal tumors predicts cancer metabolism and patient prognosis. Cancer Sci 105: 1100-1108, 2014 